\title{
ESTUDIO COMPARATIVO DE LOS COMPONENTES DE LA CALIDAD DE VIDA LABORAL
}

\author{
COMPARATIVE STUDY OF THE COMPONENTS OF QUALITY OF WORKING LIFE \\ Nélida del Carmen Castellano \\ Facultad de Ciencias Económicas \\ Universidad Nacional de Córdoba \\ Córdoba, Córdoba, Argentina \\ nelicast@gmail.com \\ Esteban Damián López \\ Facultad de Ciencias Económicas \\ Universidad Nacional de Córdoba \\ Córdoba, Córdoba, Argentina \\ amian.lopez@quappro.com

\begin{abstract}
Fecha de recepción: 28/08/2020 - Fecha de revisión: 07/09/2020 - Fecha de revisión: 06/10/2020 - Fecha de aprobación: 15/12/2020

DOI: https://doi.org/10.36995/j.visiondefuturo.2021.25.01.003.es
\end{abstract}

\section{RESUMEN}

Nuestra línea de investigación en 17 años, ha sido la Calidad Total y sus problemáticas en Pymes de Córdoba. Hasta ahora, no habíamos estudiado el concepto de Calidad de Vida Laboral (CVL).

Este proyecto forma parte de uno mayor que integra el Programa Retos Poblacionales Actuales, donde hemos incluido la CVL, pues entendemos que un reto poblacional actual es estudiar los factores que la afectan: desempleo, subempleo, precarización laboral, crisis económica, cuestiones de género que no han sido superadas en años, preconceptos sobre la menor capacidad de decisión femenina frente a la masculina, entre otros. En estos últimos meses, un factor adicional, impensado y grave, como es la cuarentena por el Covid-19, ha venido a complejizar aún más el trabajo de las personas y por ende a este concepto.

El estudio de la CVL es un campo joven, donde los autores no acuerdan idénticas dimensiones y variables para su medición. Como primera etapa, hemos comparado estudios actualizados y agregamos factores por impacto de la Calidad Total y de la cuarentena por Covid-19, para construir un modelo superador útil para la toma de decisiones y un cuestionario, el que hemos probado en algunas organizaciones.

PALABRAS CLAVE: Calidad de vida laboral; Procesos dinámicos; Calidad total; Mejora Continua; Teletrabajo.

\section{ABSTRACT}

Our line of research in 17 years has been total quality and its problems in SMEs of Córdoba. Until now, we had not studied the concept of Quality of Working Life (QWL). This

"Visión de Futuro" Año 18, Volumen No 25 Nº 1, Enero - Junio 2021 - Pág 75 -90

URL de la Revista: http://visiondefuturo.fce.unam.edu.ar/index.php/visiondefuturo/index

URL del Documento: https://visiondefuturo.fce.unam.edu.ar/index.php/visiondefuturo/issue/view/19

ISSN 1668 - 8708 - Versión en Línea

E-mail: revistacientifica@fce.unam.edu.ar

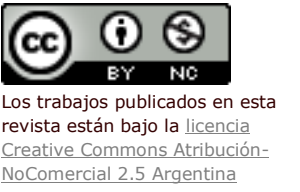


project is part of a larger one that integrates the Current Population Challenges program, where we have included the QWL, because we understand that a current population challenge is to study the factors that affect it: unemployment, underemployment, precarization, economic crisis, gender issues that have not been surpassed in years, preconceptions about the lower capacity of female decision-making against the male, among others. In recent months, an additional, unthinkable and serious factor, such as Covid-19 quarantine, has come to further complement people's work and thus this concept. The QWL study is a young field, where authors do not agree on identical dimensions and variables for measurement. As a first stage, we have compared updated studies and added factors due to the impact of total quality and Covid-19 quarantine, for building a superior model for decision-making and a questionnaire which we have probed in some organizations.

KEY WORDS: Quality of working life; Dynamic processes; Total quality; Continuous Improvement; Teleworking.

\section{INTRODUCCIÓN}

Este proyecto integra el Programa Retos Poblacionales Actuales, donde hemos incluido a la Calidad de Vida Laboral (CVL), pues entendemos que un reto poblacional actual es estudiar los factores que la afectan: desempleo, subempleo, precarización laboral, crisis económica, cuestiones de género que no han sido superadas en años, preconceptos sobre la menor capacidad de decisión femenina frente a la masculina, entre otros. En estos últimos meses, un factor adicional, impensado y grave, como es la pandemia del Covid-19, ha venido a complejizar aún más el trabajo de las personas y por ende a este concepto.

Cuando se buscan antecedentes sobre la CVL, se encuentran numerosos estudios e investigaciones, con relevamientos de datos en el ámbito de organizaciones de ramos específicos (hay numerosas del sector salud), que tienen especial interés en utilizar el concepto de la CVL de sus colaboradores para la difusión de su buena gestión. Es un campo de estudio joven, en el cual los diversos autores no acuerdan cuáles son las dimensiones y variables adecuadas para la medición de la CVL, por lo que debimos analizar varios modelos operacionales y completarlos con otras dimensiones, para que sea de real utilidad en la toma de decisiones de los directivos de las organizaciones.

"Visión de Futuro" Año 18, Volumen Nº 25 N 1, Enero - Junio 2021 - Pág 75 -90

URL de la Revista: http://visiondefuturo.fce.unam.edu.ar/index.php/visiondefuturo/index

URL del Documento: https://visiondefuturo.fce.unam.edu.ar/index.php/visiondefuturo/issue/view/19

ISSN 1668 - 8708 - Versión en Línea

E-mail: revistacientifica@fce.unam.edu.ar 
Consideramos a este proyecto como la primera parte de otro que tendrá un alcance mayor y que abordará también las diferencias de género en la CVL las cuales aún no han sido solucionadas.

\section{Preguntas de investigación}

¿Cuántas dimensiones de estudio presenta la Calidad de Vida Laboral?

¿Cuáles son los factores o componentes principales que determinan la Calidad de Vida Laboral? ¿Tienen todos los mismos pesos relativos?

¿Qué impactos producen en la Calidad de Vida Laboral los cambios ocurridos en los procesos dinámicos por la Calidad Total, en especial por la Mejora Continua?

¿Hay cambios en el trabajo de las personas originados por la cuarentena del Covid-19 y cómo afectan a la Calidad de Vida Laboral?

\section{Problema de investigación}

De las preguntas anteriores consideradas como disparadoras de nuestras inquietudes para esta indagación, es posible extraer una pregunta más comprensiva para presentarla como el problema de la investigación, que se expone a continuación:

¿Cuáles son las dimensiones y los factores o componentes que mejor explican y miden la Calidad de Vida Laboral y qué otros componentes han surgido por los cambios en los Procesos Dinámicos (Mejora Continua) y por la cuarentena del Covid-19?

\section{Objetivo General}

Estudiar las dimensiones y los factores o componentes que mejor explican y miden la Calidad de Vida Laboral. Además, deseamos conocer qué nuevos factores han surgido por los cambios en los Procesos Dinámicos (Mejora Continua) y por la cuarentena del Covid-19, para elaborar un modelo superador.

\section{Objetivos Específicos}

- Indagar las dimensiones y los factores o componentes que determinan la Calidad de Vida Laboral.

\footnotetext{
"Visión de Futuro" Año 18, Volumen N² 25 N 1, Enero - Junio 2021 - Pág 75 -90

URL de la Revista: http://visiondefuturo.fce.unam.edu.ar/index.php/visiondefuturo/index

URL del Documento: https://visiondefuturo.fce.unam.edu.ar/index.php/visiondefuturo/issue/view/19

ISSN 1668 - 8708 - Versión en Línea

E-mail: revistacientifica@fce.unam.edu.ar
} 
- $\quad$ Conocer los impactos que producen en la Calidad de Vida Laboral los cambios ocurridos en los Procesos Dinámicos, por ejemplo la Mejora Continua.

- Identificar los nuevos factores que afectan el trabajo de las personas originados en la cuarentena por Covid-19.

\section{DESARROLLO}

\section{A. Marco Conceptual}

\section{Concepto de Calidad de Vida Laboral (CVL)}

La expresión Calidad de Vida Laboral (CVL) tuvo origen en una serie de conferencias patrocinadas al final de los '60 y comienzos de los '70 por el Ministerio de Trabajo de los EE.UU. y la Fundación FORD, para hacer frente a los movimientos obreros frente a la deshumanización de las relaciones laborales. El estudio de la CVL se ha venido abordando bajo dos grandes perspectivas teórico-metodológicas: la calidad de vida del entorno de trabajo y la perspectiva de la CVL psicológica.

La CVL tiene por objetivo determinar los aspectos del trabajo que inciden en la calidad de vida de una persona, por eso se debe tener en cuenta el trabajo en sus múltiples expresiones en el ser y hacer de una persona que van más allá del salario, por tanto, se puede afirmar que es de carácter multidimensional, además, implica la reproducción de la sociedad, la construcción de identidad y sentido de pertenencia, la posibilidad de reconocimiento y satisfacción social.

En el debate internacional, la calidad del empleo y el trabajo decente ganaron importancia a partir de los '90, en especial por parte de instituciones como la OIT y NU. (Weller y Roethlisberger, 2011). La OIT en 2012, definió que el trabajo es fuente de dignidad personal, estabilidad familiar, paz en la comunidad, democracias que actúan en beneficio de todos, y crecimiento económico, que aumenta las oportunidades de trabajo productivo y el desarrollo de las empresas.

\section{Impacto de la Calidad Total en la Disciplina Administrativa}

La Calidad Total o Gestión de Calidad Total (TQM) aplicada especialmente en los '90 ha traído profundos cambios a la disciplina administrativa. Ninguna otra filosofía o metodología gerencial impactó tan fuertemente a la Administración como lo hizo la Calidad Total. Este tema ha sido uno de nuestros objetos de estudio, elaboración de artículos y exposición en eventos,

\footnotetext{
"Visión de Futuro" Año 18, Volumen Nº 25 No 1, Enero - Junio 2021 - Pág 75 -90

URL de la Revista: http://visiondefuturo.fce.unam.edu.ar/index.php/visiondefuturo/index

URL del Documento: https://visiondefuturo.fce.unam.edu.ar/index.php/visiondefuturo/issue/view/19

ISSN 1668 - 8708 - Versión en Línea

E-mail: revistacientifica@fce.unam.edu.ar
} 
desde el año 2008 en adelante.

Es una temática cuyo marco teórico toma conceptos de autores de Administración y de Gestión de Calidad Total, tales como: Hill, Ch. y Jones, G. (2011), Evans, J. y Lindsay, W. (2005), Fea, U. (1995), Pfeffer, J. (1995), Riccardi, R. (1993) y Fernández, J. A. (2005). También suma conceptos incorporando autores de Comportamiento Organizacional, entre otros: Chiavenato, I. (2007), Davis, K. y Newstrom, J. (2003), Gore, E. (2006) y Robbins, S.P. (2017). La mayoría de estas obras han sido actualizadas por sus autores en los últimos años, por ser de permanente consulta.

Se destacan los siguientes grupos de cambios ocasionados por la filosofía de la Calidad Total en la Administración, los cuales impactan directamente en la Calidad de Vida Laboral como veremos más adelante:

1. Cambios en la Estructura de las organizaciones

2. Cambios en las Funciones del proceso administrativo

3. Cambios en los elementos de la Estrategia

4. Cambios en los Procesos Dinámicos

\section{Cambios producidos en los Procesos Dinámicos}

Para este trabajo nos interesa profundizar en las variables que moldean la conducta humana (estudiadas por el behaviorismo) denominados Procesos Dinámicos. Éstos se refieren a los fluidos que circulan por la estructura de una organización, tales como: liderazgo, motivación, conflicto y comunicación. A partir de la Gestión de Calidad Total, todos ellos se han visto modificados con el objetivo superior de generar la Calidad de Vida Laboral. Los principales procesos dinámicos identificados son los siguientes:

- Liderazgo (Autoridad y delegación)

- Motivación

- Conflicto

- Comunicación

- Información

- Capacitación permanente

- Aprendizaje continuo

El liderazgo ha tenido que adecuarse al trabajo en equipos donde la conducción difiere sustancialmente del manejo de los individuos, quienes podían ser divididos para conseguir los

"Visión de Futuro" Año 18, Volumen Nº 25 No 1, Enero - Junio 2021 - Pág 75 -90

URL de la Revista: http://visiondefuturo.fce.unam.edu.ar/index.php/visiondefuturo/index

URL del Documento: https://visiondefuturo.fce.unam.edu.ar/index.php/visiondefuturo/issue/view/19

ISSN 1668 - 8708 - Versión en Línea

E-mail: revistacientifica@fce.unam.edu.ar 
objetivos del líder. La sinergia generada en los equipos de alto rendimiento conformados para cumplimentar los principios de la Gestión de Calidad Total, supera al conocimiento de cada persona y aún al mismo líder. El liderazgo debe adaptarse a los nuevos estilos de conducción, tales como la participación y el involucramiento de todo el personal en pos del objetivo superior de la satisfacción del cliente. En la actualidad se habla del Liderazgo Lean, que es aquél necesario para conducir los procesos en los cuales se ha aplicado la metodología Lean (manufactura, oficinas y servicios) (U. Dombrowski, T. Mielke, 2014).

La motivación, compañera inseparable del liderazgo, tiene que seguir las nuevas pautas de la Calidad Total y ser muy transparente y genuina. Kaoru Ishikawa siempre decía en los cursos a gerentes que cuando la Calidad entra a una organización, se terminan el engaño y la mentira. Las necesidades humanas que juegan sobre la motivación en la organización son numerosas y el método más eficaz de análisis (pero también el más difícil) debe fundarse en la comunicación entre los diferentes compañeros: los cuadros (niveles jerárquicos) deben saber escuchar y abrir un diálogo con los empleados. La verdadera Mejora Continua debe tener lugar en la tarea básica diaria y en todos los departamentos. Tiene que resultar de una cultura de mejora. La mejora diaria tiene que llegar a ser un comportamiento natural de todos los empleados y dirigidos por la voluntad de desarrollo personal y sentido de logro y no por ventajas monetarias, es un factor de motivación superior.

El conflicto ya no es evitado ni ocultado, sino que se requiere su tratamiento estratégico para eliminar sus causas, o bien, una vez producido llegar a una solución consensuada utilizando, dentro de lo posible, las técnicas de la negociación que tienden a obtener resultados del tipo ganar / ganar, luego de conocer profundamente las necesidades de las partes en conflicto.

La comunicación ahora es multidireccional y no solamente descendente, como se afirmó durante muchos años. Se propician también las comunicaciones ascendentes y laterales, es decir entre pares con quienes se conforman los equipos de trabajo. El proceso de comunicación implica conocer a los interlocutores y estar a su escucha, lo cual no es posible sin un buen clima interno; de ello se desprende que el clima interno y la comunicación son muy interdependientes. Además, la comunicación debe establecerse en un espíritu de sinceridad y de transparencia total, con un objetivo claro y conocido por todos.

La información de interés para todos los integrantes de la organización debe circular natural y libremente, a partir de la implantación de la Gestión de Calidad Total se acaban los

"Visión de Futuro" Año 18, Volumen Nº 25 No 1, Enero - Junio 2021 - Pág 75 -90

URL de la Revista: http://visiondefuturo.fce.unam.edu.ar/index.php/visiondefuturo/index

URL del Documento: https://visiondefuturo.fce.unam.edu.ar/index.php/visiondefuturo/issue/view/19

ISSN 1668 - 8708 - Versión en Línea

E-mail: revistacientifica@fce.unam.edu.ar 
secretos y los objetivos y estrategias implícitos, que sólo existen en la mente del dueño o directivo. La difusión de la información se torna una función obligatoria para los cuadros jerárquicos.

La capacitación permanente surge como consecuencia de un cambio cultural, donde se otorga gran importancia a la capacitación del personal en todos sus estamentos. El significado ulterior es que la capacitación no tiene fin, siempre se debe aprender conocimientos y habilidades nuevas. El objetivo del sistema es usado también para evaluar el desarrollo del empleado. Antes de que construyamos autos, nosotros construimos personas, esta frase proverbial de Toyota ilustra muy bien la gran importancia del desarrollo del empleado. Por ello, el desarrollo del empleado debe estar en alta posición en los objetivos del sistema. El proceso sólo puede ser tan bueno como el empleado. Por esta razón, ambos deben ser desarrollados por igual. (U. Dombrowski, T. Mielke, 2014).

El aprendizaje continuo es otra expresión del cambio cultural, que transforma a los empleados en seres profundamente interesados en todos los procesos de la organización y en su mejora continua (Kaizen), con una permanente curiosidad en las actividades que los rodean y en la posibilidad de cambio y optimización. El aprendizaje debe tener lugar en ciclos cortos. La solución de problemas y el aprendizaje deben realizarse de forma estandarizada y basada en el ciclo PDCA o PHEA (Planear, Hacer, Evaluar y Actuar). Son esenciales numerosas repeticiones regulares y una aproximación de experimento científico en las diversas tareas. (U. Dombrowski, T. Mielke, 2014).

En la Era Industrial, las organizaciones que tenían éxito eran las que incrementaban su capital financiero, el cual hacía que crecieran y se expandieran. En cambio hoy, las organizaciones exitosas son extremadamente ágiles e innovadoras. Vivimos en la Era de la Información y el capital financiero deja lugar a los activos intangibles e invisibles, llamados Capital Intelectual. Uno de los principales componentes del Capital Intelectual es el Capital Humano, o sea el capital constituido por gente, por talentos y por competencias. El capital físico se deprecia con el uso, pero el valor del conocimiento aumenta cada día más; el caudal de conocimiento de una persona no disminuye, se complementa con el de los otros, por eso adquieren tanta importancia los procesos dinámicos de capacitación permanente y aprendizaje continuo, como rasgos de una cultura organizacional democrática e impulsora.

Riccardi (1993), un pionero de los estudios gerenciales en Argentina, defendía que la Dirección por Calidad Total debe aplicar la Gestión de Calidad Total a procesos y productos,

"Visión de Futuro" Año 18, Volumen No 25 N 1, Enero - Junio 2021 - Pág 75 -90

URL de la Revista: http://visiondefuturo.fce.unam.edu.ar/index.php/visiondefuturo/index

URL del Documento: https://visiondefuturo.fce.unam.edu.ar/index.php/visiondefuturo/issue/view/19

ISSN 1668 - 8708 - Versión en Línea

E-mail: revistacientifica@fce.unam.edu.ar 
pero también a los recursos humanos y al ambiente de trabajo, para generar la Calidad de Vida de Trabajo, sin la cual no se logra la primera, y dejó ya en esos años, instalado definitivamente el concepto de Calidad de Vida Laboral.

Pfeffer (1995) dejó en claro su opinión sobre la importancia de la gente en la organización y afirmó que tanto la Gestión de Calidad como la Gestión del Cambio, sólo pueden alcanzarse con éxito a través de los recursos humanos. Este pensamiento revela la mayor intención de reconocimiento para los trabajadores. Cuenta entre sus seguidores a prestigiosos autores como: Stephen Robbins, Martha Alles, Andrew Dubrin, Enrique Franklin y Mario Krieger. El reconocimiento es uno de los factores o componentes principales de la Calidad de Vida Laboral.

\section{B. Estado del Arte}

Hemos revisado varias publicaciones actuales que tratan de determinar los componentes o factores que impactan en la Calidad de Vida Laboral y establecer una medición. Entre estas publicaciones, hemos seleccionado las que nos brindaron aportes al marco conceptual y a la metodología de medición, las que presentamos en la sección Desarrollo.

\section{Metodología}

En este proyecto aplicaremos como metodología, el Estudio y Análisis Comparativo de los diversos modelos y esquemas que hemos relevado de la bibliografía seleccionada, que es específica y actualizada.

En la sección Desarrollo, mencionamos las publicaciones seleccionadas por su interés en los aportes al marco conceptual, a las nuevas condiciones o componentes considerados para el estudio de la CVL y a los modelos que presentan, considerando la naturaleza multidimensional del concepto de CVL.

En ese estudio comparativo, agregamos también las condiciones y componentes originados en los impactos de la Calidad Total (Mejora Continua) en los Procesos Dinámicos de las organizaciones, los que han tenido como objetivo superior, la mayor Calidad de Vida Laboral para todos los trabajadores. Asimismo, consideramos los efectos de la cuarentena por Covid-19 en el trabajo de las personas y por ende, en la Calidad de Vida Laboral.

\footnotetext{
"Visión de Futuro" Año 18, Volumen N² 25 N 1, Enero - Junio 2021 - Pág 75 -90

URL de la Revista: http://visiondefuturo.fce.unam.edu.ar/index.php/visiondefuturo/index

URL del Documento: https://visiondefuturo.fce.unam.edu.ar/index.php/visiondefuturo/issue/view/19

ISSN 1668 - 8708 - Versión en Línea

E-mail: revistacientifica@fce.unam.edu.ar
} 
De esta forma, elaboramos un modelo superador e integrador lo más completo posible, por la mayor cantidad y calidad de los factores o componentes de la CVL, que sea útil para la toma de decisiones.

A partir de dicho modelo integrador, construimos un cuestionario digital conteniendo las dimensiones indicadas anteriormente y realizamos una prueba piloto del mismo para determinar el nivel de comprensión de las preguntas, el que resultó satisfactorio.

A partir del marco teórico de los Cambios en los Procesos Dinámicos, producidos por la Calidad Total, realizamos un cuadro con las dimensiones y variables identificadas, similar al de otros autores.

De las publicaciones seleccionadas tomamos los cuadros más completos con los cuales efectuamos el análisis comparativo, con el fin de integrar la mayor cantidad de dimensiones en un modelo superador para indagar y medir adecuadamente el complejo concepto de la Calidad de Vida Laboral. También agregamos una publicación sobre los efectos de la cuarentena por Covid-19 en el trabajo de las personas.

\section{Aportes de las publicaciones seleccionadas:}

a) González-Hidalgo-Salazar-Preciado (2010): Elaboración y Validación del Instrumento para Medir Calidad de Vida en el Trabajo "CVT-GOHISALO. Los autores han realizado lo que dice el título del artículo, en un instrumento de 7 dimensiones y 74 variables para medir la CVT. Este modelo ha sido ampliamente utilizado hasta la actualidad, por estar validado y ser muy completo. Sin embargo, la extensión del instrumento (74 ítems) se ha presentado siempre como un inconveniente, lo que llevó a otros investigadores Pando, González, Aranda y Elizalde (2018) a la elaboración de una versión breve (31 ítems), la que también fue validada.

b) Granados, Isabel (2011): Calidad de Vida Laboral: Historia, Dimensiones y Beneficios. La autora ha realizado una recopilación teórica de la historia de la calidad de vida laboral, así como de los diversos conceptos que se han elaborado en torno a ella, revisando los trabajos más destacados. El estudio de la calidad de vida laboral se ha venido abordando básicamente bajo dos grandes perspectivas teórico-metodológicas: la calidad de vida del entorno de trabajo y la perspectiva de la CVL psicológica. La perspectiva de la calidad del entorno de trabajo, persigue el objetivo de conseguir mejorar la calidad de vida mediante el logro de los intereses organizacionales. En cambio, la perspectiva de la calidad de vida laboral psicológica muestra mayor interés por el trabajador. Las variables estudiadas se clasifican en: Condiciones "Visión de Futuro" Año 18, Volumen Nº 25 N 1, Enero - Junio 2021 - Pág 75 -90 URL de la Revista: http://visiondefuturo.fce.unam.edu.ar/index.php/visiondefuturo/index URL del Documento: https://visiondefuturo.fce.unam.edu.ar/index.php/visiondefuturo/issue/view/19 ISSN 1668 - 8708 - Versión en Línea

E-mail: revistacientifica@fce.unam.edu.ar 
Objetivas: vienen dadas por el análisis de variables referidas al medioambiente físico, tecnológico, contractual y productivo. Mientras que las Condiciones Subjetivas refieren a la esfera privada y mundo laboral, organización y función directiva y beneficios.

c) Martínez-Oviedo-Luna (2013): Condiciones de trabajo que impactan en la vida laboral. Estudiaron las variables de condiciones de trabajo que impactan en la calidad de vida laboral, relevando publicaciones a lo largo de 20 años. Proponen un esquema de clasificación conceptual (ECC) de los factores de condiciones de trabajo: 1. Condiciones Ambientales: involucra aspectos como la satisfacción laboral, los riesgos laborales, la carga de trabajo, entre otros. 2. Condiciones Ergonómicas: relacionadas con el sitio de trabajo donde el empleado desarrolla sus actividades y el bienestar que le ofrece. 3 . Condiciones Económicas: encaminadas a la percepción del trabajador con respecto al salario recibido.

Además mencionan: el clima psicosocial y la CVL e incluyen factores como la conciliación de vida laboral y familiar, implicación laboral y estado de ánimo. Y finalmente: igualdad de género, seguridad y salud en el trabajo, flexibilidad en el trabajo, formación y posibilidades de promoción, organización de la jornada laboral y la vida personal, diversidad y no discriminación. d) Becerra Gualdrón (2015): Calidad de Empleo con Enfoque de Género: Propuesta de un Marco Conceptual. La autora afirma que el análisis de la CVL con enfoque de género tiene un abordaje escaso, por eso presenta un marco conceptual muy completo para su análisis.

Resultan muy útiles los datos aportados por los autores mencionados en la prolija revisión realizada, siendo el más importante el de Weller y Rothlisberger (2011) de CEPAL, con las dimensiones para América Latina. Finalmente, la autora propone una medición de la CVL basada en dicho trabajo y elabora un cuestionario con dos partes: para la empresa y para los individuos, el cual se basa tanto en percepciones subjetivas como en datos objetivos, atendiendo a la multidimensionalidad del concepto de CVL.

e) Cruz Velazco (2018): La Calidad de Vida Laboral y el Estudio del Recurso Humano: Una Reflexión Sobre su Relación con las Variables Organizacionales. Quiere determinar las principales variables de la CVL. Realizó la búsqueda en artículos científicos publicados en los últimos 7 años de los términos: CVL, variables organizacionales, CVL y variables, CVL y relaciones, revisando las bases de datos más reconocidas. De ese análisis obtuvo tablas con relaciones entre las variables independientes y dependientes que afectan la CVL. Concluye en

"Visión de Futuro" Año 18, Volumen Nº 25 N 1, Enero - Junio 2021 - Pág 75 -90

URL de la Revista: http://visiondefuturo.fce.unam.edu.ar/index.php/visiondefuturo/index

URL del Documento: https://visiondefuturo.fce.unam.edu.ar/index.php/visiondefuturo/issue/view/19

ISSN 1668 - 8708 - Versión en Línea

E-mail: revistacientifica@fce.unam.edu.ar 
que, por la variedad de desafíos para el bienestar de los trabajadores, se necesita un enfoque integrado de la investigación que pueda ayudar a mejorar la calidad de vida laboral percibida.

f) Esbry, G. (04/06/2020): Teletrabajo: Nuevo desafío para el mundo laboral. La Voz del Interior. Córdoba. Argentina. La cuarentena por el Covid-19 nos ha obligado a cambiar muchas de nuestras costumbres cotidianas entre las que se encuentra el trabajo; lo hacemos desde el hogar con la modalidad teletrabajo, también llamada home-office. Según la Encuesta de Indicadores Laborales (EIL) del Ministerio de Trabajo de la Nación, antes de la pandemia, menos del 8\% de los trabajadores lo hacía en forma remota, un 70,4\% eran varones jóvenes empleados de Servicios Tecnológicos. Ahora más del 40\% lo hace, alcanzando picos del $85 \%$. Otro dato interesante lo muestra la Red Internacional de Educación para el Trabajo (RIET), el $63 \%$ de los que hicieron teletrabajo desearían continuar con esta forma.

El Teletrabajo presenta pros y contras tanto para las empresas como para los trabajadores; para las primeras se abaratan costos y se promueve mayor productividad, pero se complica la capacidad de supervisión y coordinación. Para los segundos, se reducen los tiempos y costos del transporte y se mejora el equilibrio entre vida laboral y personal, pero es difícil regular los horarios de trabajo y contar con las herramientas informáticas necesarias.

Si el teletrabajo queda instalado como una modalidad extendida quizás no baste con una ley que garantice el cumplimiento de las obligaciones y de los derechos laborales, también será necesario pensar en una nueva cultura del trabajo que aborde de manera integral las relaciones entre empresas y empleados, y en la que el hogar, la familia y la tecnología tendrán un protagonismo mayor que el que hasta ahora mostraban.

\section{Cuadro comparativo de modelos operacionales}

A partir de la revisión de trabajos realizada tomamos el modelo CVT-GOHISALO-2010 como base por estar validado y tener numerosos antecedentes de su utilización en estudios sobre la CVL.

Lo comparamos con los modelos operacionales propuestos por los autores Granados y Becerra (Tabla № 1), para identificar las coincidencias entre los factores de cada modelo. Asimismo, y como era objetivo del presente trabajo, se incluyeron en la comparación los Procesos Dinámicos (Tabla № 2).

\footnotetext{
"Visión de Futuro" Año 18, Volumen N² 25 N 1, Enero - Junio 2021 - Pág 75 -90

URL de la Revista: http://visiondefuturo.fce.unam.edu.ar/index.php/visiondefuturo/index

URL del Documento: https://visiondefuturo.fce.unam.edu.ar/index.php/visiondefuturo/issue/view/19

ISSN 1668 - 8708 - Versión en Línea

E-mail: revistacientifica@fce.unam.edu.ar
} 
Tabla № 1: CVT-GOHISALO-2010 vs. Modelos Operacionales

\begin{tabular}{c|l|c|c|c|}
\hline \multicolumn{2}{|c|}{} & $\begin{array}{c}\text { Variables del } \\
\text { modelo }\end{array}$ & $\begin{array}{c}\text { Variables } \\
\text { incluidas (1) }\end{array}$ & $\begin{array}{c}\text { Variables } \\
\text { excluidas (2) }\end{array}$ \\
$\begin{array}{c}\text { Modelos } \\
\text { Operacionales }\end{array}$ & Granados & 21 & 10 & 11 \\
\cline { 2 - 5 } & Becerra & 28 & 12 & 16 \\
\hline
\end{tabular}

(1) Cantidad de variables incluidas en CVT-GOHISALO-2010

(2) Cantidad de variables excluidas en CVT-GOHISALO-2010

Fuente: Elaboración Propia

Tabla № 2: CVT-GOHISALO-2010 vs. Procesos Dinámicos

\begin{tabular}{|c|l|c|}
\hline & Liderazgo & $\begin{array}{c}\text { Variables incluidas } \\
\text { (1) }\end{array}$ \\
\cline { 2 - 3 } & Motivación & 12 \\
\hline \multirow{2}{*}{$\begin{array}{c}\text { Procesos } \\
\text { Dinámicos }\end{array}$} & Conflicto & 13 \\
\cline { 2 - 3 } & Comunicación & 2 \\
\cline { 2 - 3 } & Información & 4 \\
\hline Capacitación & 2 \\
\hline & Aprendizaje & 1 \\
\hline
\end{tabular}

(1) Cantidad de variables referidas a procesos dinámicos incluidas en CVT-GOHISALO-2010

Fuente: Elaboración Propia

Con el objetivo de alcanzar un modelo operacional superador e integrador de la mayor parte de las dimensiones identificadas como relevantes para el estudio de la CVL, decidimos utilizar el modelo CVT-GOHISALO-2018 (Breve), completado con una dimensión referida a la Mejora Continua y otra sobre el impacto de la cuarentena por Covid-19 en el trabajo de las personas. (Tabla № 3).

Realizamos una prueba piloto del instrumento resultante con el fin de evaluar la comprensión de las preguntas formuladas.

\section{CONCLUSIONES}

1. El Análisis Comparativo permitió ampliar nuestro conocimiento teórico sobre la CVL:

- El concepto de la CVL es eminentemente multidimensional y no existe consenso sobre cuáles son las dimensiones que lo definen.

\footnotetext{
"Visión de Futuro" Año 18, Volumen No 25 N 1, Enero - Junio 2021 - Pág 75 -90

URL de la Revista: http://visiondefuturo.fce.unam.edu.ar/index.php/visiondefuturo/index

URL del Documento: https://visiondefuturo.fce.unam.edu.ar/index.php/visiondefuturo/issue/view/19

ISSN 1668 - 8708 - Versión en Línea

E-mail: revistacientifica@fce.unam.edu.ar
} 
- No es materialmente posible realizar un modelo que incluya la totalidad de dimensiones identificadas por los autores.

- CVT-GOHISALO-2010 es el modelo que ha sido validado y ampliamente utilizado en numerosas investigaciones sobre CVL, pero tiene el inconveniente de su extensión (74 componentes). Por ello, otros investigadores han elaborado una versión breve del mismo.

- La incorporación de todos los factores de procesos dinámicos y del impacto de la cuarentena por Covid-19 en el modelo operacional, lo tornaría demasiado extenso.

2. Por ello fue posible elaborar una transferencia hacia un cuestionario lo más completo posible para investigar y medir la CVL:

- Construimos un modelo operacional basado en CVT-GOHISALO-2018 (Breve) e incorporamos: una dimensión sobre la Mejora Continua, compuesta por los factores de capacitación permanente y aprendizaje continuo y una dimensión para evaluar el impacto de la cuarentena por Covid-19, en el trabajo de las personas. (Tabla № 3).

- Confiamos que este modelo superador sea útil para la toma de decisiones de los directivos.

- El cuestionario resultante del modelo integral se sometió a una prueba piloto para evaluar su comprensión por parte de los encuestados.

Tabla № 3: Cuestionario (Modelo Integral Superador de Calidad de Vida Laboral)

\begin{tabular}{|c|l|}
\hline № & \multicolumn{1}{c|}{ Pregunta } \\
\hline 1 & Proceso que se sigue para supervisar mi trabajo \\
\hline 2 & Trato que recibo de mis superiores \\
\hline 3 & Se me ha indicado de manera clara y precisa la forma en que debo de hacer mi trabajo \\
\hline 4 & $\begin{array}{l}\text { Recibo retroalimentación por parte de mis compañeros y superiores en cuanto a la } \\
\text { evaluación que hacen de mi trabajo }\end{array}$ \\
\hline 5 & $\begin{array}{l}\text { En mi organización se reconocen los esfuerzos de eficiencia y preparación con } \\
\text { oportunidades de promoción }\end{array}$ \\
\hline 6 & $\begin{array}{l}\text { Considero que tengo libertad para expresar mis opiniones en cuanto al trabajo sin temor a } \\
\text { represalias de mis jefes }\end{array}$ \\
\hline 7 & La forma en que están diseñados los procedimientos para realizar mi trabajo \\
\hline 8 & El salario que tengo \\
\hline 9 & Las condiciones físicas de mi área laboral (ruido, iluminación, limpieza, orden, etc.) \\
\hline 10 & El tipo de capacitación que recibo por parte de la organización \\
\hline 11 & $\begin{array}{l}\text { Considero que recibo en cantidad suficiente los insumos necesarios para la realización de } \\
\text { mis actividades laborales }\end{array}$ \\
\hline 12 & Trato que tengo con mis compañeros de trabajo \\
\hline 13 & En mi organización se respetan mis derechos laborales \\
\hline 14 & $\begin{array}{l}\text { Busco los mecanismos para quitar los obstáculos que identifico en el logro de mis objetivos } \\
\text { y metas de trabajo }\end{array}$ \\
\hline 15 & La forma de contratación, con que cuento en este momento \\
\hline
\end{tabular}

"Visión de Futuro" Año 18, Volumen Nº 25 N 1, Enero - Junio 2021 - Pág 75 -90

URL de la Revista: http://visiondefuturo.fce.unam.edu.ar/index.php/visiondefuturo/index

URL del Documento: https://visiondefuturo.fce.unam.edu.ar/index.php/visiondefuturo/issue/view/19

ISSN 1668 - 8708 - Versión en Línea

E-mail: revistacientifica@fce.unam.edu.ar 


\begin{tabular}{|c|c|}
\hline № & Pregunta \\
\hline 16 & Trabajar en esta organización (comparando con otras organizaciones que conozco) \\
\hline 17 & Las funciones que desempeño en esta organización \\
\hline 18 & El uso que hago en este trabajo de mis habilidades y potenciales \\
\hline 19 & El reconocimiento que recibo de otras personas por mi trabajo \\
\hline 20 & Mi desempeño como profesional en este trabajo \\
\hline 21 & La calidad de los servicios básicos de mi vivienda \\
\hline 22 & ¿Qué tanto percibo que mi trabajo es útil para otras personas? \\
\hline 23 & $\begin{array}{l}\text { Cuento con la integridad de mis capacidades físicas, mentales y sociales para el } \\
\text { desempeño de mis actividades diarias (vestir, caminar, trasladarse, alimentarse, etc.) }\end{array}$ \\
\hline 24 & Mi trabajo me permite acceder en cantidad y calidad a mis alimentos \\
\hline 25 & Mi trabajo contribuye con la buena imagen que tiene la organización ante sus usuarios \\
\hline 26 & El logro de mis objetivos con respecto al trabajo \\
\hline 27 & $\begin{array}{l}\text { Considero que el logro de satisfactores personales que he alcanzado se deben a mi trabajo } \\
\text { en la organización }\end{array}$ \\
\hline 28 & Mis potencialidades mejoran por estar en este trabajo \\
\hline 29 & $\begin{array}{l}\text { Considero que el trabajo me ha permitido brindar el cuidado necesario para conservar la } \\
\text { integridad de mis capacidades físicas, mentales y sociales }\end{array}$ \\
\hline 30 & $\begin{array}{l}\text { Mi trabajo me permite cumplir con las actividades que planeo para cuando estoy fuera del } \\
\text { horario de trabajo }\end{array}$ \\
\hline 31 & Mis actividades laborales me dan oportunidad de convivir con mi familia \\
\hline 32 & Se realizan capacitaciones en mi organización \\
\hline 33 & $\begin{array}{l}\text { La capacitación que recibo de mi organización sobre: DETECCIÓN DE FALLAS es } \\
\text { suficiente }\end{array}$ \\
\hline 34 & La capacitación que recibo de mi organización sobre: GESTIÓN DE CALIDAD es suficiente \\
\hline 35 & $\begin{array}{l}\text { La capacitación que recibo de mi organización sobre: RESOLUCIÓN DE PROBLEMAS es } \\
\text { suficiente }\end{array}$ \\
\hline 36 & En mi organización, se gestionan las necesidades y requerimientos de los clientes \\
\hline 37 & En mi organización, se gestionan las no conformidades \\
\hline 38 & Me interesa aprender más sobre mi tarea \\
\hline 39 & $\begin{array}{l}\text { Me interesa aprender más sobre la interrelación de mis tareas con otros procesos de la } \\
\text { organización }\end{array}$ \\
\hline 40 & Me interesa aprender más sobre la filosofía del Kaizen (Mejora Continua) \\
\hline 41 & ¿En qué medida la cuarentena por COVID-19 afectó el desarrollo de su trabajo? \\
\hline 42 & $\begin{array}{l}\text { ¿En este contexto de cuarentena, realizó teletrabajo o home office para el desarrollo de sus } \\
\text { tareas? }\end{array}$ \\
\hline 43 & ¿Cuál es su GRADO DE SATISFACCIÓN en relación al Teletrabajo? \\
\hline
\end{tabular}

\section{Líneas de investigación futuras}

Como una etapa posterior a este proyecto, se prevé la aplicación del cuestionario que ya ha sido probado, para el estudio de la CVL en las organizaciones de la Ciudad de Córdoba. Se ha previsto trabajar con una muestra del padrón de empresas del Ministerio de la Producción de la Provincia de Córdoba, seleccionando las que están localizadas en nuestra ciudad.

\footnotetext{
"Visión de Futuro" Año 18, Volumen N² 25 N 1, Enero - Junio 2021 - Pág 75 -90

URL de la Revista: http://visiondefuturo.fce.unam.edu.ar/index.php/visiondefuturo/index

URL del Documento: https://visiondefuturo.fce.unam.edu.ar/index.php/visiondefuturo/issue/view/19

ISSN 1668 - 8708 - Versión en Línea

E-mail: revistacientifica@fce.unam.edu.ar
} 


\section{REFERENCIAS}

Becerra Gualdrón, J. (2015): Calidad de Empleo con Enfoque de Género: Propuesta de un Marco Conceptual. Universidad Pedagógica y Tecnológica de Colombia (Colombia).

Autor (2008): Gestión de Calidad Total: Cambios en los Procesos Dinámicos de las Organizaciones. $17^{\circ}$ Congreso Nacional de Profesionales en Ciencias Económicas.

Autor (2008): Gestión de Calidad Total, Asociación Cooperadora FCE, Córdoba.

Chiavenato, I. (2007): Administración de recursos humanos. El capital humano de las organizaciones, $8^{a}$ Edición, McGraw-Hill, México.

Cruz Velazco, J. E. (2018): La calidad de vida laboral y el estudio del recurso humano: una reflexión sobre su relación con las variables organizacionales. Pensamiento \& Gestión, núm. 45, Fundación Universidad del Norte - Barranquilla, Colombia.

Davis, k. y Newstrom, J. (2003): Comportamiento Humano en el Trabajo. Comportamiento Organizacional, 10ª Edición, McGraw Hill, México.

Dombrowski, U. y Mielke, T. (2014): Variety Management in Manufacturing. Proceedings of the 47th CIRP Conference on Manufacturing Systems, Procedia CIRP 17 (2014) 565 - 570.

Evans, J. y Lindsay, W. (2005): Administración y Control de la Calidad. Thompson International, 6를 Edición, México.

Fea, U. (1995): Competitividad es Calidad Total. Manual para salir de la crisis y generar empleo, Alfaomega Marcombo, México.

Fernández, J. A. (2005): Lecciones de El Arte de la Guerra, Revista Gestión, Vol. 10, № 1, Pág. 94-99.

González R., Hidalgo G., Salazar J., Preciado M. (2010): Elaboración y Validación del Instrumento para Medir Calidad de Vida en el Trabajo CVT-GOHISALO. Ciencia \& Trabajo. Año 12, | Número 36.

Gore, E. (2006): Aprendizaje y Organización, Ediciones Granica, Buenos Aires.

Granados, I. (2011): Calidad de Vida Laboral: Historia, Dimensiones y Beneficios. Revista II PSI, Facultad de Psicología, Universidad Mayor San Marcos, Perú.

Hernandez Sampieri, R., Fernández Collado, C. y Baptista Lucio, M. (2010): Metodología de la Investigación, McGraw-Hill. México.

Hill, Ch. y Jones, G. (2011): Administración Estratégica. Un enfoque integrado. 9a Edición. Cengage Learning. México.

\footnotetext{
"Visión de Futuro" Año 18, Volumen No 25 N 1, Enero - Junio 2021 - Pág 75 -90

URL de la Revista: http://visiondefuturo.fce.unam.edu.ar/index.php/visiondefuturo/index

URL del Documento: https://visiondefuturo.fce.unam.edu.ar/index.php/visiondefuturo/issue/view/19

ISSN 1668 - 8708 - Versión en Línea

E-mail: revistacientifica@fce.unam.edu.ar
} 
Martínez L., Oviedo O. y Luna C. (2013): Condiciones de trabajo que impactan en la vida laboral. Salud Uninorte, vol. 29, núm. 3, 2013, pp. 542-560. Universidad del Norte. Barranquilla, Colombia.

Pfeffer, J. (1995): Competitive Advantage through People, Harvard Business School Press, U.S.A.

Riccardi, R. (1993): La Cultura de la Calidad Total, 3ª Edición, Ediciones Fausto. Buenos Aires.

Robbins, S.P. (2017): Comportamiento Organizacional. Conceptos, Controversias y Aplicaciones. 17ª Edición. Pearson. México.

Saravia Sánchez, F. (Coord.) (2013). Métodos de Investigación Social y de la Empresa. Editorial Pirámide. Madrid.

Uriel, E. y Aldas, J. (2005): Análisis multivariante aplicado. Madrid. Thomson-Paraninfo.

\section{RESUMEN BIOGRÁFICO}

\section{Nélida del Carmen Castellano}

Profesora Titular por Concurso D.E. - Profesora Consulta UNC desde 2018. Dpto. de Administración, Facultad de Ciencias Económicas UNC. Categoría I Programa Docente/Investigador. Evaluadora de Proyectos de Investigación Universidades Nacionales. Evaluadora en CONEAU de carreras de posgrado. Evaluadora Internacional Premio Iberoamericano de la Calidad (FUNDIBEQ). Directora del Centro de Transferencia Calidad Total. Consultora en temas de Calidad Total y de Administración.

\section{Esteban Damián López}

Licenciado en Administración - FCE - UNC. Cursando la Maestría en Estadística Aplicada - UNC. Fundador de Quappro - Consultora especializada en Sistemas de Gestión de la Calidad. Profesor y Responsable de Tecnología Educativa en Fundación Carbonell. Docente Universitario de la materia Organización y Gestión de Instituciones de Salud en el Instituto Universitario de Ciencias Biomédicas de Córdoba (IUCBC) y de Introducción a la Matemática - FRC - UTN. Integrante del Centro de Transferencia en Calidad Total - FCE - UNC: ha trabajado en asistencia técnica a organizaciones públicas y privadas y en proyectos de investigación referidos a la Gestión de Calidad Total. Responsable de Calidad de Servicio del Hospital Privado Universitario de Córdoba (2012-2016).

\footnotetext{
"Visión de Futuro" Año 18, Volumen N 25 N 1, Enero - Junio 2021 - Pág 75 -90

URL de la Revista: http://visiondefuturo.fce.unam.edu.ar/index.php/visiondefuturo/index

URL del Documento: https://visiondefuturo.fce.unam.edu.ar/index.php/visiondefuturo/issue/view/19

ISSN 1668 - 8708 - Versión en Línea

E-mail: revistacientifica@fce.unam.edu.ar
} 